\title{
Striped Patterns in Radially Driven Suspensions with Open Boundaries
}

\author{
Mahdieh Mohammadi, ${ }^{1,2}$ Maniya Maleki,, , Æ Adam Wysocki, ${ }^{3}$ and M. Reza Shaebani ${ }^{3, \text { 丹 }}$ \\ ${ }^{1}$ Department of Physics \& Optics Research Center, \\ Institute for Advanced Studies in Basic Sciences, Zanjan 45137-66731, Iran \\ ${ }^{2}$ Institute of Physics, Otto von Guericke University Magdeburg, 39106 Magdeburg, Germany \\ ${ }^{3}$ Department of Theoretical Physics \& Center for Biophysics, \\ Saarland University, 66123 Saarbrücken, Germany
}

\begin{abstract}
We study the motion of radially driven fluid-immersed particles in a novel Hele-Shaw cell with open boundaries. The initially uniform suspension forms a striped pattern within a specific range of horizontal oscillation frequencies and for sufficiently large amplitudes. We observe that the initial coarsening dynamics of the stripes gradually slows down and the pattern reaches a steady state after a few minutes. The distance between the stripes in the steady state exhibits an exponentially saturating increase with increased oscillation amplitude or frequency. The width of the stripes decreases as a power-law with the frequency while its amplitude dependence follows a logistic function. We propose a mechanism - based on the interplay between shear stress, hydrodynamic interactions, and frictional forces - to link the structural characteristics of the stripes to the properties of the oscillatory external drive.
\end{abstract}

Formation of elongated patterns is ubiquitously observed in a wide variety of horizontally driven granular systems. Examples range from ripples in sandy deserts and along many coasts to striped patterns in colloids, suspensions, dry granular media, and nanoparticle composites [1 14]. Under horizontal shaking or oscillatory excitation, various types of instabilities may arise in dry granular media and suspensions. This can lead to, e.g., separation of different species [15], shear-induced segregation [16 18] in environments with a nonuniformly distributed shear strain [19, 20], or, more frequently, stripe formation 8 [12, 21 27].

Although identifying the criteria under which the stripes emerge has been the focus of many studies, the underlying mechanisms have not been fully understood to date; one cannot reliably predict whether stripes emerge in a given granular system under a certain external drive. The influential factors on formation and stability of structures in dry granular media are frictional contacts $26-$ 29] and inelastic collisions which lead to effective (longrange) interactions 30 32. In colloids and suspensions, hydrodynamic forces and viscous drag also play a crucial role [5, 10, 33]. While the origin of stripe formation in granular mixtures is often attributed to the differences between the components $8-11,21,25]$, stripes form even between identical particles [4, 5, 26]. The role of lateral walls in stripe formation [12, 26, 34] and whether the coarsening of the patterns leads eventually to phase separation [4, 10, 35, 36] have also been under debate.

The detailed structural characteristics of the stripessuch as the distance between them (hereafter called their wavelength $\lambda$ ) or their width $w$ - are even less understood. $w$ was reported to grow with the amplitude $\mathcal{A}$ of the oscillatory drive in suspensions [10]. Although there is a consensus that the increase of $\mathcal{A}$ enhances $\lambda$ in ripples and stripes, different $\lambda-\mathcal{A}$ dependencies have been proposed so far: While a linear increase of $\lambda$ with $\mathcal{A}$ is often reported-e.g. in granular mixtures 10, 21, sand ripples under water [37], suspensions [12, 38], and even for immersed particles in a capillary tube [7] -, other dependencies of $\lambda$ on $\mathcal{A}$ - such as a power-law relation with an exponent smaller than one in suspensions [33] or a faster than linear increase in sand ripples [39] - were also reported. For the frequency dependence of $\lambda$, various contradicting relations can be found in the literature: A power-law decay of $\lambda$ with $f$ (though with different exponents) has been reported for sand ripples [40], heavy beads immersed in water [33], and binary granular mixtures 22]. In contrast, an increase of the form $\lambda \sim f^{2}$ in granular mixtures [10], a growth faster than $f^{2}$ in sand ripples [39], and an increase and gradual saturation in granular mixtures 21] have been reported as well. $\lambda$ was even found to be independent of the frequency of the oscillatory drive in suspensions 12,38$]$. In the absence of a unified theoretical framework, it is generally not clear how the interplay between the external drive and the nature of interparticle and particle-wall interactions dictates $w$ and $\lambda$. A nonuniform drive - as, e.g., exerted by propagating waves in curved coasts - makes the problem

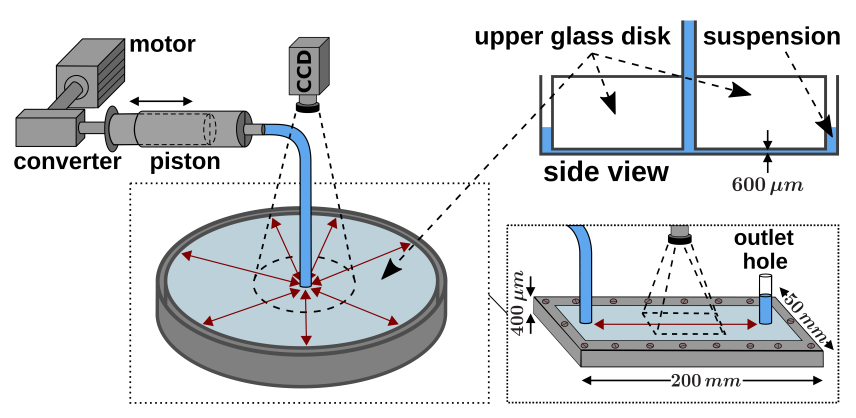

FIG. 1. Sketch of the experimental setup. The right inset represents the alternative rectangular setup. The double-headed red arrows indicate the direction of oscillation. 
even more complicated.

Here, we introduce a novel Hele-Shaw cell in which a suspension of a single type of particles is radially driven under open and periodic boundary conditions in the radial and angular directions, respectively (Fig.11). The new setup mimics the boundary conditions in curved coasts and also enables us to study a broad range of horizontal oscillation amplitudes simultaneously. The emergence of curved striped patterns in this setup proves that the lateral walls perpendicular to the driving direction are not required for stripe formation. We characterize the structure by analyzing the radial-dependence of $w$ and $\lambda$ and show that the growth of both quantities with $\mathcal{A}$ ultimately saturates at high driving amplitudes. We propose a self-amplification mechanism for the evolution of $w$ which results in a logistic growth versus $\mathcal{A}$, as observed experimentally. By considering the interplay between streaming flow fields, shear stress exerted by the nonuniformly driven fluid, and interparticle and particle-wall frictional forces, we propose a mechanism which eventually stops the coarsening process and accounts for the observed relations between the structural characteristics of the stripes and the properties of the oscillatory drive.

Setup. - Our experimental system consists of a hollow cylindrical plexiglass container with a diameter of $R=300 \mathrm{~mm}$. A concentric thick plexiglass disk with a smaller diameter of $285 \mathrm{~mm}$ is placed at a distance of $600 \mu \mathrm{m}$ from the bottom plate. An inlet hole is created in the center of the glass disk and is connected through a soft silicone tube and a syringe to a mechanical converter, which transforms the rotational motion of an AC motor into a sinusoidal vibration (Fig.1). The periodic motion of the converter is transferred to the syringe to induce a back and forth motion of the suspension inside the tube and cell. The suspension is radially driven in the spacing between the parallel glass surfaces at the bottom and is exposed to the open air at the lateral gap between the cylinder and the disk. The oscillation frequency $f$ is controlled by an AC speed controller and the amplitude of the piston is held constant at $21 \mathrm{~mm}$, which results in a radial fluid displacement of, e.g., $\mathcal{A} \simeq 10 \mathrm{~mm}$ at the distance $10 \mathrm{~mm}$ from the axis. Besides this main setup, a second rectangular setup is also used for complementary experiments; see Fig.11(inset) and [4].

The cell is filled with a granular suspension composed of spherical glass beads with density $\rho=2.5 \mathrm{~g} / \mathrm{cm}^{3}$ and diameter in the range $\sigma=150-200 \mu \mathrm{m}$ immersed in ethanol (with density $\rho_{\mathrm{eth}}=0.8 \mathrm{~g} / \mathrm{cm}^{3}$ and dynamic viscosity $\eta=1.2 \mathrm{mPa} \cdot \mathrm{s}$ at $20^{\circ} \mathrm{C}$ ) or water. We used salt $(\mathrm{NaCl})$ and dishwashing liquid in aqueous suspensions to avoid electrostatic effects and for an easier sedimentation of the beads. Although similar patterns form in both cases, here we present the results of the ethanol suspension. The maximum particle Reynolds number is around $\mathrm{Re} \approx 2$ and the flow generated by the bead is not instantaneous and time-reversible. The beads occupy a volume fraction of around $7 \%$, which corresponds to a mean interparticle distance of $\sim 300 \mu \mathrm{m}$. We use a Canon Rebel T2i camera with a resolution of $110 \mu \mathrm{m} /$ pixel and frame rate of $24 \mathrm{fps}$ to image the suspension from above. To characterize the structural properties of the patterns, the contrast between the solution and the beads is increased by placing the setup on a dark plate and illuminating from above. In the circular setup, the widths of
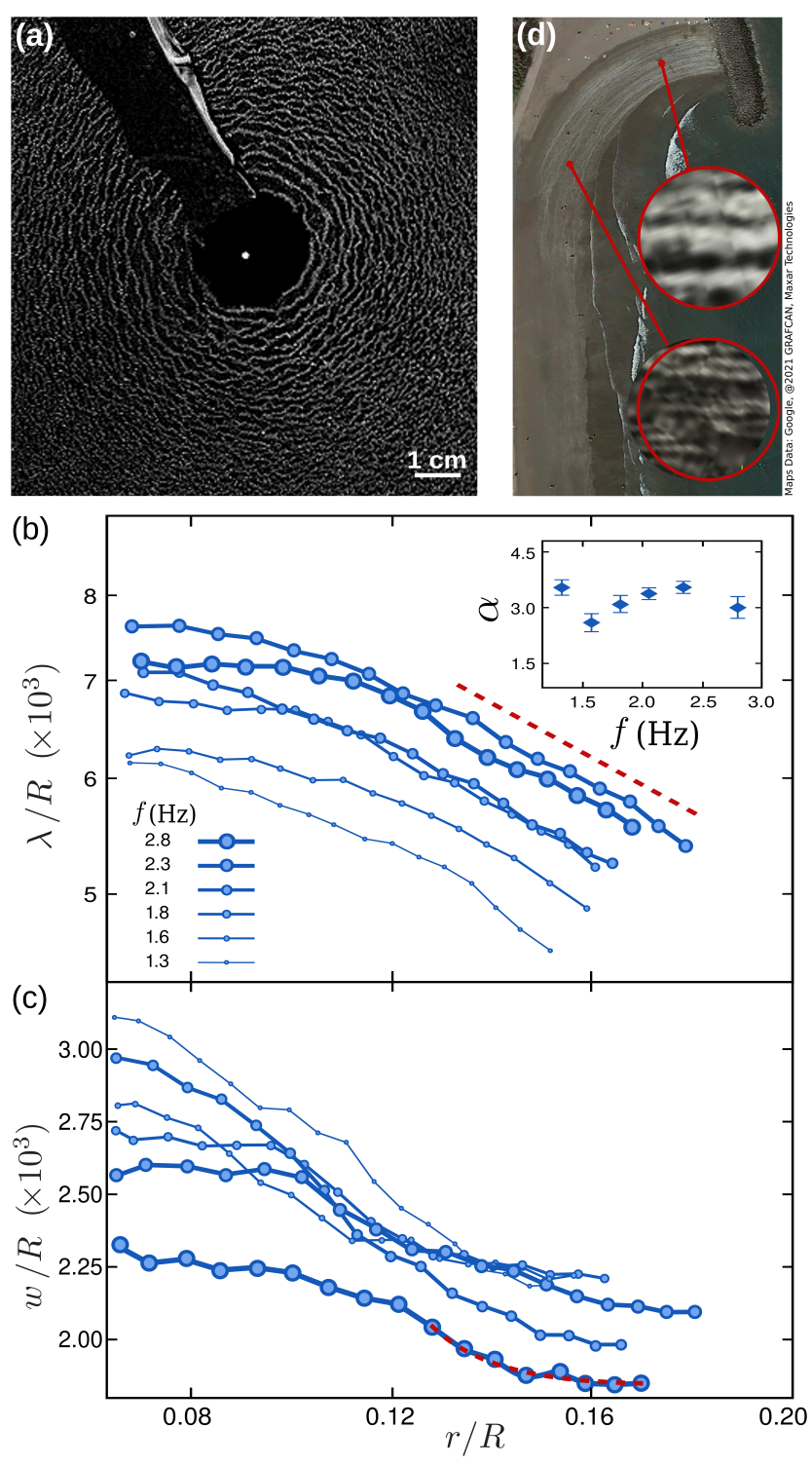

FIG. 2. (a) Representative image of the steady stripes in a suspension vibrated at $f=2.1 \mathrm{~Hz}$. (b) $\lambda$, scaled by the radius of curvature $R$ at the open boundary, vs $r / R$ in log-lin scale. The dashed guide line represents an exponential decay. Inset: Slope of the exponential tail vs $f$. (c) $w$ versus $r / R$. The dashed line shows a $1 / r$ decay as a guide to the eye. (d) Satellite image of a sandy beach near $27^{\circ} 45^{\prime} \mathrm{N}, 15^{\circ} 33^{\prime} \mathrm{W}$ (image credit: Google Maps), showing a clear difference in the ripple pattern along neighboring curved or flat coast lines. 
successive stripes and the spacing between them along differently oriented radii are measured and a radial coordinate $r$ is assigned to the center of each recorded stripe width or spacing between two neighboring stripes. Next, by binning the radial distance, the results belonging to the same bin are averaged over all orientations to obtain $w(r)$ and $\lambda(r)$. In the rectangular setup, the measurement is performed along parallel lines oriented along the oscillation direction and the mean $w$ and $\lambda$ are calculated over the entire data.

Formation of patterns. - The initially well-mixed suspension becomes unstable within a few seconds and subsequently elongated curved structures emerge for a sufficiently large amplitude of the piston (practically $>5 \mathrm{~mm}$ ) and within the frequency range $1-3 \mathrm{~Hz}$, for which the driving strength $\Gamma \propto f^{2}$ is neither too weak (no pattern) nor too strong (vortex formation). We do not aim here to precisely identify the stripe formation subdomain in the $f-\mathcal{A}$ space. The pattern gradually coarsens but reaches a dynamical saturation after a few minutes, where the overall shape of the stripes becomes time invariant [4]. Notably, we have observed striped patterns even in a setup where the distance between the plates is slightly larger than the size of one single bead. Also we would like to emphasize that no visual sign of vortices or turbulent flow has been observed since the analyzed region $(10 \mathrm{~mm}<r<60 \mathrm{~mm})$ is far away from both boundaries. Indeed, vortices may form near the inlet pipe. The size $L$ of the affected region is given by $L / D=0.39 \operatorname{Re}_{i}^{1 / 3}(H / D)^{4 / 5}$, where $D=6 \mathrm{~mm}$ is the inlet diameter, $H=0.6 \mathrm{~mm}$ the gap height between the two plates, and $R e_{i} \approx 400$ the maximal inlet flow Reynolds number [41]. Accordingly, the vortex length cannot be larger than $L \approx 3 \mathrm{~mm}$ in our experiment. Furthermore, $R e_{i}$ as well as the flow Reynolds number between the plates $(R e \approx 6)$ are much smaller than the critical Reynolds number $R e_{c} \approx 2040$ for the onset of turbulence in pipe flows [42]. It can be shown that the velocity profile between the plates in our setup is a quasi-parabolic profile in the vertical dimension and there are small deviations from a time-periodic plane Poiseuille flow. Nevertheless, the laminar radial flow is preserved and the system is far away from the high Reynolds number and turbulent flow regime.

Figure2(a) shows a typical striped pattern in the steady state. Both the width $w$ of the stripes and spacing $\lambda$ between them grow as approaching the center of the cell, until they ultimately saturate; see Fig.2(b),(c). The tail of $\lambda$ decays almost exponentially with the radial coordinate $r$, i.e. $\lambda \sim \exp \left[-\alpha \frac{r}{R}\right]$ with a $f$-independent decay rate, $\alpha$ as shown in the inset of Fig.2(b). The $\lambda-r$ curves shift to higher wavelengths with increasing $f$ but converge at high frequencies. The tail of $w-r$ curves decays relatively slower and can be approximated as $w \sim 1 / r$. With a further reduction of $\lambda$ towards the open boundary, the pattern gradually becomes indistinguishable and the suspension remains uniform in the limit $r \rightarrow R$. Interestingly, curvature-dependent patterns can be also observed in sand ripples formed along curved coasts [see e.g. Fig.2(d)]: Because of the curvature of the coast line, the incoming plane waves are transformed into curved oscillatory wave fronts that fade away towards the open boundary at the shore. Sediment transport is a dominant process in ripple formation, which plays no role in stripe formation in our well-mixed suspensions; nevertheless, the analogies between the two systems (esp. the radial drive and open boundaries) and between the emerging patterns suggest that the global scales should be irrelevant in the formation of ripples and the mechanism is governed by the local scales, without a need for effective long-range interactions (as it is demonstrated in the following for the stripes in our driven suspensions). A detailed analysis and comparison between the underlying physical mechanisms and emerging patterns in these systems will be presented elsewhere.

Dependence of $\lambda$ on $\mathcal{A}$ and $f$. - To better understand the influence of the oscillation amplitude on the distance between the stripes, we plot $\lambda$ vs $\mathcal{A}(\propto 1 / r$ since the local displacement of the fluid in the circular setup decays with the inverse radial distance from the center). $\lambda$ increases with $\mathcal{A}$ with a decreasing rate and gradually saturates to a $f$-dependent saturation wavelength $\lambda_{\infty, f}$. When scaled by $\lambda_{\infty, f}$, the data for all $f$ values lie on a master curve

$$
\lambda=\lambda_{\infty, f}\left(1-\mathrm{e}^{-\mathcal{A} / \mathcal{A}_{\mathrm{c}}}\right),
$$

with $\mathcal{A}_{\mathrm{c}} \simeq 2.8 \pm 0.2 \mathrm{~mm}$ being the characteristic saturation amplitude, i.e. a measure of how fast the maximum possible spacing $\lambda_{\infty, f}$ is reached. The fit curve in the main panel of Fig. 3(a) is obtained by fitting the entire data for all frequencies to the exponential convergence Eq. (1) using a single fit parameter $\mathcal{A}_{\mathrm{c}}$. Moreover, by repeating the fitting procedure for each frequency, we separately obtain $\mathcal{A}_{\mathrm{c}}$ for each frequency. The results presented in the inset of Fig.3(a) show that $\mathcal{A}_{\mathrm{c}}$ remains nearly unchanged with respect to $f . \lambda_{\infty, f}$ is the maximum possible spacing between the stripes at a given frequency. The motility range of the stripes (thus the spacing $\lambda$ between them) increases with the fluid motion amplitude until $\lambda$ approaches the upper limit $\lambda_{\infty, f}$. At larger driving amplitudes, the stripe motion cannot follow the oscillatory flow anymore. $\lambda_{\infty, f}$ grows with $f$ and eventually saturates at higher frequencies. We also average the results over the entire range of $r$ - i.e. over all oscillation amplitudesat each frequency to clarify the overall dependence of $\lambda$ on $f$. Figure 3(b) shows that the behavior of the averaged wavelength $\langle\lambda\rangle$ can be described by an exponential convergence

$$
\langle\lambda\rangle=\langle\lambda\rangle_{\infty}\left(1-\mathrm{e}^{-f / f_{\mathrm{c}}}\right),
$$

where $\langle\lambda\rangle_{\infty}$ is the saturation wavelength and $f_{\mathrm{c}}$ the characteristic frequency. A similar behavior of $\lambda$ vs $f$ was numerically observed in dry granular systems [21]. 

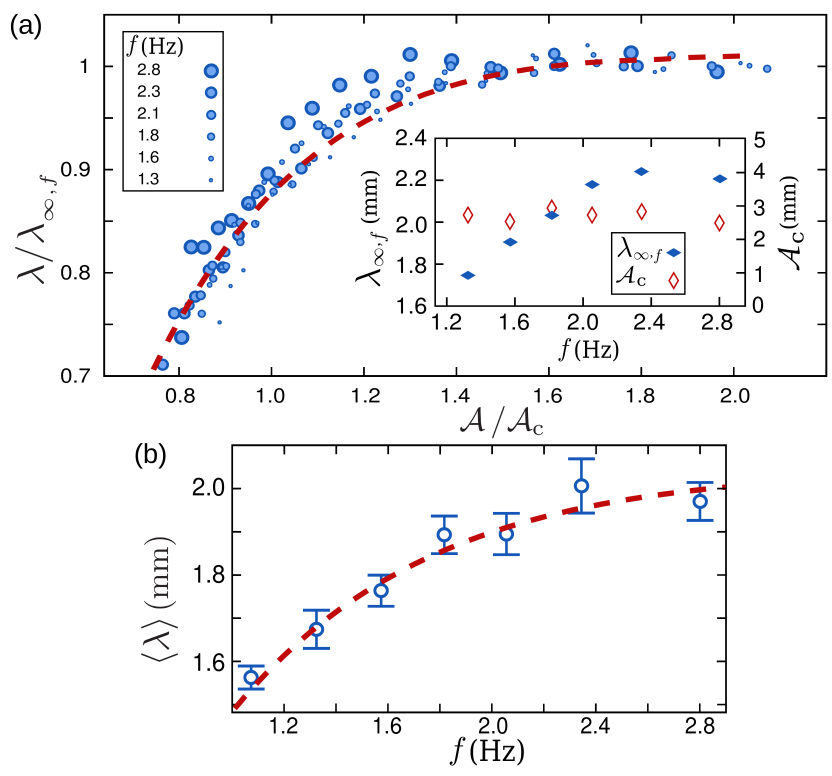

FIG. 3. (a) $\lambda$, scaled by $\lambda_{\infty, f}$, vs $\mathcal{A} / \mathcal{A}_{\mathrm{c}}$ at different values of $f$. The line is a fit to the exponential convergence Eq. (1). Inset: $\lambda_{\infty, f}$ and $\mathcal{A}_{\mathrm{c}}$ vs $f$. (b) Averaged wavelength over all oscillation amplitudes vs $f$. The line is a fit to Eq. (2).

Effects of the oscillatory drive on w.- At a given $f, w$ grows monotonically with increasing $\mathcal{A}$ towards the center of the setup. The growth rate increases with $\mathcal{A}$ far from the center, but then decreases and ultimately decays to zero at large amplitudes (i.e. in the vicinity of the axis) [Fig. 4 (a)]. The saturation width $w_{\infty, f}$ is thinner at higher frequencies. The characteristic saturation amplitude $\mathcal{A}_{\mathrm{s}}$ is slightly larger compared to $\mathcal{A}_{\mathrm{c}}$ for $\lambda-\mathcal{A}$ behavior, but it is similarly independent of $f$.

Next, we average the width over the entire range of $r$ at each frequency. The resulting averaged width $\langle w\rangle$

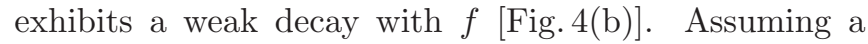
power-law decay, we obtain an exponent $0.3 \leq \beta \leq 0.5$ but the $r$-averaged data is not conclusive and it is difficult to detect a weak dependency on $f$ because the patterns practically emerge over different ranges of $r$ with varying $f$, the frequency window of stripe formation in this setup is too narrow, and $w$ is of the order of just a few particle diameters. Therefore, we repeat the experiments in the rectangular setup shown in Fig.1 which enables us to observe the striped pattern over a broader range of $f$ and analyze the $w-f$ relation more precisely. We present the results of experiments with polystyrene beads in the inset of Fig.4(b). It turns out that $w$ decays as a power-law

$$
w \sim f^{-\beta},
$$

with $\beta=0.51 \pm 0.08$. Note that only a region of size $6 \mathrm{~cm} \times 2 \mathrm{~cm}$ (along and perpendicular to the vibration direction, respectively) around the center line of the rectangular cell is considered (i.e. far from the boundaries), where the flow field remains uniform.
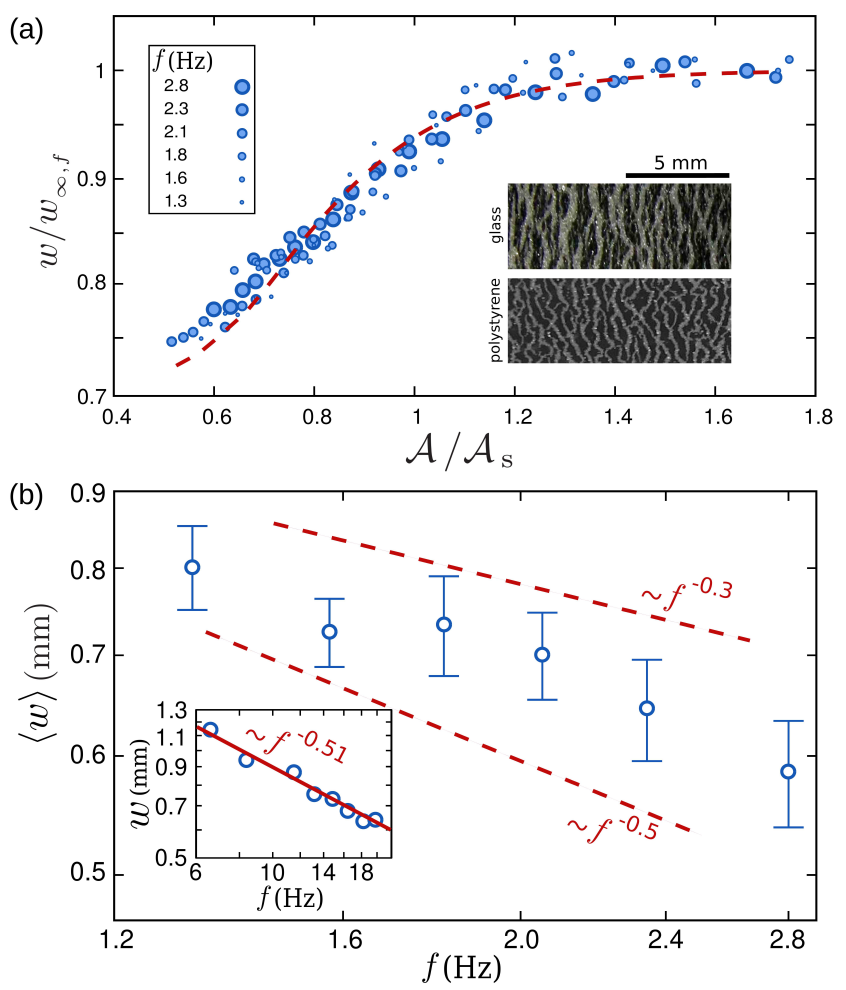

FIG. 4. (a) $w$, scaled by $w_{\infty, f}$, vs $\mathcal{A} / \mathcal{A}_{\mathrm{s}}$ at different $f$. The dashed line is the logistic form given by Eq. (4). Insets: Comparison between the stripes formed by glass or polystyrene beads at the same driving strength in the rectangular setup. (b) Averaged stripe width over all oscillation amplitudes versus $f$ in logarithmic scales. The lines represent power-law decays as a guide to the eye. Inset: $w$ vs $f$ for experiments in the rectangular setup. The line is a power-law fit, Eq. (3).

Stripe formation mechanism. - Our proposed mechanism is built upon a few major underlying physical processes: (i) A critical driving is necessary to generate irreversible collisions between the particles, which induce diffusive motion in an otherwise non-Brownian system 43 47]; it is known that noise is an important driver of phase separation [48]. (ii) A closer look at the steadystate structures reveals that the particles are in physical contact with each other and also with the bottom surface. Such frictional structures are hyperstatic [49, 50] and resist against perturbations [51]; the extent of the contact network enhances the stability of the structure. (iii) On the contrary, the shear stress $\tau$ induced by the nonuniformly driven fluid tends to break the contacts; $\tau$ is stronger at a higher $f$. (iv) The oscillatory motion of a particle in the fluid generates a streaming flow field around the particle with a penetration depth $\delta \sim 1 / \sqrt{f}$ $[5,53,52,53]$; this vorticity pattern sucks or ejects the fluid towards or away from the particle, which induces repulsive (attractive) interactions along (perpendicular to) the vibration direction. The superposition of such hydrodynamic interactions was shown to create elongated 
structures perpendicular to the vibration direction [5].

By vibrating the initially well-mixed suspension, particles collide randomly due to slight differences between their actual displacement amplitudes and due to differences in the velocity of particles moving at different heights in a Poiseuille flow, which leads to the formation of frictional contacts. On the other hand, contacts may break due to oscillatory shear forces. At a sufficiently high particle density and for a moderate driving strength, the rate of contact formation surpasses the breakage rate, which results in the nucleation of small units. The interactions between these units through their streaming flow fields cause elongated structures, as explained above. The stripes coarsen by random merging of smaller units or individual colliding particles. Nevertheless, the dynamics of the structures is limited to the local oscillation amplitude of the fluid, thus, the coarsening process is short ranged. Upon increasing the stripe width, a weak hydrodynamic repulsion gradually develops between the neighboring stripes in the vibration direction, which eventually arranges the stripes at distances $\lambda \propto \mathcal{A}$. However, $\lambda$ does not continue to grow with $\mathcal{A}$ at an extremely large $\mathcal{A}$ because the weak hydrodynamic repulsion cannot push the stripes over long distances. In this regime, $\lambda$ and $w$ grow until the contact area between the stripe and the bottom plate becomes large enough that the stripe-wall frictional force ultimately balances the shear force which tends to slide the stripe over the surface. This leads to the saturation distance $\lambda_{\infty}$ between the stripes. The empirical observation Eq. (1) implies that the rate of increasing $\lambda$ with respect to $\mathcal{A}$ is proportional to the distance from the saturation wavelength, i.e. $\frac{\mathrm{d} \lambda}{\mathrm{d} \mathcal{A}} \propto\left(\lambda_{\infty}-\lambda\right)$.

Increasing $f$ has a twofold effect: First, it strengthens $\tau$ against the interparticle friction. This enhances the contact breakage rate, which reduces $w$; second, it also strengthens $\tau$ against the particle-wall friction such that the balance between them is reached at a larger $\lambda$, where the stripe becomes sufficiently thick. Nevertheless, we observe that $\lambda$ finally saturates with $f$. An object of size $w$ can follow the oscillatory fluid only if the characteristic time of the fluid motion $t_{\mathrm{f}} \sim 1 / f$ is larger than the relaxation time $t_{\mathrm{r}}$ of the object in the fluid (which scales as $t_{\mathrm{r}} \sim w^{2}$ in low Reynolds number regime [54]). This sets an upper limit on $f$ for a given object size, above which the shear cannot efficiently move the object. We obtain $f_{\max } \approx 4 \mathrm{~Hz}$ for a stripe of width $w=3 \sigma$, which is consistent with the frequency window $1 \mathrm{~Hz}<f<3 \mathrm{~Hz}$ for pattern formation in our circular setup (For $f>3 \mathrm{~Hz}$, we observe that no striped pattern forms, vortices appear, and the laminar radial flow is not preserved anymore). Also, synchronization of two timescales at the steady state results in $w \sim 1 / \sqrt{f}$, in agreement with the scaling relation Eq. (3).

According to the proposed scenario, decreasing the friction coefficient between the particles destabilizes the structures against shear forces and, thus, leads to thinner stripes. We confirm the validity of this prediction by repeating the experiments in the rectangular setup with polystyrene beads which have a smaller friction coefficient compared to the glass beads. As shown in the inset of Fig. 4 (a) for experiments at $f=19.8 \mathrm{~Hz}$, thicker stripes form in the case of glass beads. A similar conclusion can be drawn when comparing glass and polystyrene data at other frequency values.

The growth of $w$ by random merging of smaller structures follows a self-amplification mechanism: A thicker stripe has a larger contact area, thus, a higher chance for further merging events. It also contains a wider frictional contact network which is more stable and reduces the particle loss rate by the oscillatory shear. However, the coarsening process gradually slows down as the number of remaining floating objects in the environment reduces. The available resources are bounded to the spacing between the stripes, which is controlled by $\mathcal{A}$. Hence, we propose that the growth rate of $w$ vs $\mathcal{A}$ is given by $\frac{\mathrm{d} w}{\mathrm{~d} \mathcal{A}} \propto w\left(w_{\infty}-w\right)$, thus, the amplitude dependence of the coarsening process follows a logistic growth form

$$
w=\frac{w_{\infty}}{2}\left(1+\frac{1}{1+\kappa \mathrm{e}^{-\left(\mathcal{A}-\mathcal{A}_{\min }\right) / \mathcal{A}_{\mathrm{s}}}}\right),
$$

with $\kappa=\frac{w_{\infty}}{2 w_{\min }-w_{\infty}}-1$ and $\mathcal{A}_{\min }$ being the minimum amplitude at which the patterns emerge, i.e., $w\left(\mathcal{A}=\mathcal{A}_{\min }\right) \equiv w_{\text {min }}$. Indeed, three of the model parameters can be extracted from the experimental data and there remains only a single fit parameter $\mathcal{A}_{\mathrm{s}}$. When rescaled to the maximum possible width $w_{\infty, f}$, the asymptotic value of the rescaled width $\frac{w}{w_{\infty, f}}$ fluctuates around 1 with less than $3 \%$ error for all frequencies; see Fig.4(a). Thus, we fit the entire $w / w_{\infty}$ data (i.e. for all frequencies) to Eq. (4) for simplicity. The minimum possible stripe width $w_{\min }$ is taken as the stripe width at the maximum radial distance above which the pattern is indistinguishable (corresponding to $\mathcal{A}_{\min } \simeq 1.8 \pm 0.2$ ). By fitting the experimental data to the logistic growth form, we obtain $\mathcal{A}_{\mathrm{s}} \simeq 3.6 \pm 0.2 \mathrm{~mm}$. As shown in Fig.4(a), Eq. (4) and the experimental data are in remarkable agreement. Also the leading $\mathcal{A}$-dependent term at small amplitudes is proportional to $\mathcal{A}($ i.e. $\propto 1 / r)$ in agreement with Fig.2(c).

In conclusion, we have made a step forward in understanding the mechanism of stripe formation in vibrated suspensions by considering the structures at the particle level in a novel Hele-Shaw cell. Three ingredients dictate the structure of the striped pattern: the oscillatory shear that mixes the suspension, the frictional contacts that stabilize the structures and prevent the stripes from sliding over the surface, and the streaming flow fields around the stripes that induce repulsion between the neighboring stripes while promote the merging of smaller units into the growing elongated structures. Our proposed mechanism is entirely governed by the local scales and explains 
the trends of the structural characteristics of the stripes in terms of the properties of the oscillatory external drive. Understanding the emerging hydrodynamic interactions between the structures in an oscillating fluid is still a challenging problem which deserves further detailed studies. Our results help to better understand the fundamental processes underlying widespread striped pattern formation in vibrated granular systems and can open up a new avenue for the design of more efficient technologies for separating particles from a fluid flow.

We thank Robabeh Moosavi for her help with Fig.4. M. Mohammadi acknowledges the hospitality of the group of Prof. Ralf Stannarius and partial support through the Landesstipendium Sachsen-Anhalt and Deutsche Forschungsgemeinschaft (DFG) within project STA 425/46-1. Correspondence and requests for materials should be addressed to m_maleki@iasbs.ac.ir or shaebani@lusi.uni-sb.de.

* m_maleki@iasbs.ac.ir

† shaebani@lusi.uni-sb.de

[1] B. Andreotti, P. Claudin, and O. Pouliquen, "Aeolian sand ripples: Experimental study of fully developed states," Phys. Rev. Lett. 96, 028001 (2006).

[2] Q. Zhao, C. E. Finlayson, D. R. E. Snoswell, A. Haines, C. Schäfer, P. Spahn, G. P. Hellmann, A. V. Petukhov, L. Herrmann, P. Burdet, P. A. Midgley, S. Butler, M. Mackley, Q. Guo, and J. J. Baumberg, "Large-scale ordering of nanoparticles using viscoelastic shear processing," Nat. Commun. 7, 11661 (2016).

[3] C. Reichhardt, J. Thibault, S. Papanikolaou, and C. J. O. Reichhardt, "Laning and clustering transitions in driven binary active matter systems," Phys. Rev. E 98, 022603 (2018).

[4] R. Moosavi, M. Maleki, M. R. Shaebani, J. Carlos RuizSuárez, and E. Clément, "Stripe formation in horizontally oscillating granular suspensions," EPL 107, 34006 (2014).

[5] D. Klotsa, M. R. Swift, R. M. Bowley, and P. J. King, "Chain formation of spheres in oscillatory fluid flows," Phys. Rev. E 79, 021302 (2009).

[6] T. Vissers, A. van Blaaderen, and A. Imhof, "Band formation in mixtures of oppositely charged colloids driven by an ac electric field," Phys. Rev. Lett. 106, 228303 (2011).

[7] F. Zoueshtiagh, P. J. Thomas, V. Thomy, and A. Merlen, "Micrometric granular ripple patterns in a capillary tube," Phys. Rev. Lett. 100, 054501 (2008).

[8] P. M. Reis, G. Ehrhardt, A. Stephenson, and T. Mullin, "Gases, liquids and crystals in granular segregation," EPL 66, 357-363 (2004).

[9] T. Mullin, "Coarsening of self-organized clusters in binary mixtures of particles," Phys. Rev. Lett. 84, 47414744 (2000).

[10] P. Sánchez, M. R. Swift, and P. J. King, "Stripe formation in granular mixtures due to the differential influence of drag," Phys. Rev. Lett. 93, 184302 (2004).

[11] P. M. Reis and T. Mullin, "Granular segregation as a crit- ical phenomenon," Phys. Rev. Lett. 89, 244301 (2002).

[12] Y. L. Roht, I. Ippolito, J. P. Hulin, D. Salin, and G. Gauthier, "Stripes instability of an oscillating non-brownian iso-dense suspension of spheres," EPL 121, 54002 (2018).

[13] J. Yuan and D. Wang, "Experimental investigation of total bottom shear stress for oscillatory flows over sand ripples," J. Geophys. Res. 123, 6481-6502 (2018).

[14] M. Mazzuoli, A. G. Kidanemariam, and M. Uhlmann, "Direct numerical simulations of ripples in an oscillatory flow," J. Fluid Mech. 863, 572-600 (2019).

[15] C. Lozano, I. Zuriguel, A. Garcimartín, and T. Mullin, "Granular segregation driven by particle interactions," Phys. Rev. Lett. 114, 178002 (2015).

[16] K. M. Hill and Y. Fan, "Isolating segregation mechanisms in a split-bottom cell," Phys. Rev. Lett. 101, 088001 (2008).

[17] C. Barentin, E. Azanza, and B. Pouligny, "Flow and segregation in sheared granular slurries," EPL 66, 139145 (2004).

[18] A. Garcimartin, I. Larrea, C. Lozano, and I. Zuriguel, "Cluster splitting in granular segregation driven by horizontal shaking," EPJ Web Conf. 140, 04004 (2017).

[19] R. Moosavi, M. R. Shaebani, M. Maleki, J. Török, D. E. Wolf, and W. Losert, "Coexistence and transition between shear zones in slow granular flows," Phys. Rev. Lett. 111, 148301 (2013).

[20] D. Fenistein and M. van Hecke, "Wide shear zones in granular bulk flow," Nature 425, 256 (2003).

[21] M. Pica Ciamarra, A. Coniglio, and M. Nicodemi, "Shear instabilities in granular mixtures," Phys. Rev. Lett. 94, 188001 (2005).

[22] C. M. Pooley and J. M. Yeomans, "Stripe formation in differentially forced binary systems," Phys. Rev. Lett. 93, 118001 (2004).

[23] M. Fujii, A. Awazu, and H. Nishimori, "Segregationpattern reorientation of a granular mixture on a horizontally oscillating tray," Phys. Rev. E 85, 041304 (2012).

[24] M. Pica Ciamarra, A. Coniglio, and M. Nicodemi, "Dynamically induced effective interaction in periodically driven granular mixtures," Phys. Rev. Lett. 97, 038001 (2006).

[25] A. Wysocki and H. Löwen, "Oscillatory driven colloidal binary mixtures: Axial segregation versus laning," Phys. Rev. E 79, 041408 (2009).

[26] D. Krengel, S. Strobl, A. Sack, M. Heckel, and T. Pöschel, "Pattern formation in a horizontally shaken granular submonolayer," Granular Matter 15, 377-387 (2013).

[27] R. Möbius and C. Heussinger, "(ir)reversibility in dense granular systems driven by oscillating forces," Soft Matter 10, 4806-4812 (2014).

[28] M. R. Shaebani, T. Unger, and J. Kertész, "Unjamming due to local perturbations in granular packings with and without gravity," Phys. Rev. E 78, 011308 (2008).

[29] C. Goldenberg and I. Goldhirsch, "Friction enhances elasticity in granular solids," Nature 435, 188-191 (2005).

[30] I. Zuriguel, J. F. Boudet, Y. Amarouchene, and H. Kellay, "Role of fluctuation-induced interactions in the axial segregation of granular materials," Phys. Rev. Lett. 95, 258002 (2005).

[31] M. R. Shaebani, J. Sarabadani, and D. E. Wolf, "Nonadditivity of fluctuation-induced forces in fluidized granular media," Phys. Rev. Lett. 108, 198001 (2012).

[32] M. R. Shaebani, J. Sarabadani, and D. E. Wolf, "Long- 
range interactions in randomly driven granular fluids," Phys. Rev. E 88, 022202 (2013).

[33] R. Wunenburger, V. Carrier, and Y. Garrabos, "Periodic order induced by horizontal vibrations in a twodimensional assembly of heavy beads in water," Phys. Fluids 14, 2350-2359 (2002).

[34] V. Loisel, M. Abbas, O. Masbernat, and E. Climent, "Inertia-driven particle migration and mixing in a wallbounded laminar suspension flow," Phys. Fluids 27, 123304 (2015).

[35] P. M. Reis, T. Sykes, and T. Mullin, "Phases of granular segregation in a binary mixture," Phys. Rev. E 74, 051306 (2006).

[36] D. Pihler-Puzović and T. Mullin, "The timescales of granular segregation in horizontally shaken monolayers," Proc. Roy. Soc. A 469, 20130203 (2013).

[37] A. Stegner and J. E. Wesfreid, "Dynamical evolution of sand ripples under water," Phys. Rev. E 60, R3487R3490 (1999).

[38] Y. Roht, G. Gauthier, J. Hulin, D. Salin, R. Chertcoff, H. Auradou, and I. Ippolito, "Volume fraction instability in an oscillating non-brownian iso-dense suspension," EPJ Web Conf. 140, 09029 (2017).

[39] A. A. Ivanova and V. G. Kozlov, "Sand-fluid interface under vibration," Fluid Dynamics 37, 277-293 (2002).

[40] G. Rousseaux, A. Stegner, and J. E. Wesfreid, "Wavelength selection of rolling-grain ripples in the laboratory," Phys. Rev. E 69, 031307 (2004).

[41] Y. Stergiou, K. Eckert, and K. Schwarzenberger, "Entrance effects in a radial hele-shaw cell: Numerical and experimental study," Chem. Eng. J. 428, 131146 (2022).

[42] K. Avila, D. Moxey, A. de Lozar, M. Avila, D. Barkley, and B. Hof, "The onset of turbulence in pipe flow," Science 333, 192-196 (2011).

[43] D. J. Pine, J. P. Gollub, J. F. Brady, and A. M. Leshansky, "Chaos and threshold for irreversibility in sheared suspensions," Nature 438, 997-1000 (2005).

[44] L. Corte, P. M. Chaikin, J. P. Gollub, and D. J. Pine, "Random organization in periodically driven systems," Nat. Phys. 4, 420-424 (2008).

[45] J. S. Guasto, A. S. Ross, and J. P. Gollub, "Hydrodynamic irreversibility in particle suspensions with nonuniform strain," Phys. Rev. E 81, 061401 (2010).

[46] B. Metzger, P. Pham, and J. E. Butler, "Irreversibility and chaos: Role of lubrication interactions in sheared suspensions," Phys. Rev. E 87, 052304 (2013).

[47] R. Jeanneret and D. Bartolo, "Geometrically protected reversibility in hydrodynamic loschmidt-echo experiments," Nat. commun. 5, 1-8 (2014).

[48] A. J. Bray, "Theory of phase-ordering kinetics," Adv. Phys. 51, 481-587 (2002).
[49] M. R. Shaebani, T. Unger, and J. Kertész, "Extent of force indeterminacy in packings of frictional rigid disks," Phys. Rev. E 79, 052302 (2009).

[50] T. Unger, J. Kertész, and D. E. Wolf, "Force indeterminacy in the jammed state of hard disks," Phys. Rev. Lett. 94, 178001 (2005).

[51] M. R. Shaebani, T. Unger, and J. Kertész, "Unjamming of granular packings due to local perturbations: Stability and decay of displacements," Phys. Rev. E 76, 030301 (2007).

[52] D. Klotsa, M. R. Swift, R. M. Bowley, and P. J. King, "Interaction of spheres in oscillatory fluid flows," Phys. Rev. E 76, 056314 (2007).

[53] F. Otto, E. K. Riegler, and G. A. Voth, "Measurements of the steady streaming flow around oscillating spheres using three dimensional particle tracking velocimetry," Phys. Fluids 20, 093304 (2008).

[54] J. Geng, J. Tang, W. Cai, Y. Wang, D. Jing, and L. Guo, "Periodical oscillation of particle-laden laminar flow within a tubular photocatalytic hydrogen production reactor predicted by discrete element method," Int. J. Hydrog. Energy 46, 9653-9665 (2021).

[55] I. S. Aranson and L. S. Tsimring, "Patterns and collective behavior in granular media: Theoretical concepts," Rev. Mod. Phys. 78, 641-692 (2006).

[56] T. Schnautz, R. Brito, C. A. Kruelle, and I. Rehberg, "A horizontal brazil-nut effect and its reverse," Phys. Rev. Lett. 95, 028001 (2005).

[57] G. C. M. A. Ehrhardt, A. Stephenson, and P. M. Reis, "Segregation mechanisms in a numerical model of a binary granular mixture," Phys. Rev. E 71, 041301 (2005).

[58] A. Zippelius and K. Huang, "Density-wave fronts on the brink of wet granular condensation," Sci. Rep. 7, 3613 (2017).

[59] J. E. Kollmer, T. Shreve, J. Claussen, S. Gerth, M. Salamon, N. Uhlmann, M. Schröter, and T. Pöschel, "Migrating shear bands in shaken granular matter," Phys. Rev. Lett. 125, 048001 (2020).

[60] A. Valance and V. Langlois, "Ripple formation over a sand bed submitted to a laminar shear flow," Eur. Phys. J. B 43, 283-294 (2005).

[61] G. Metcalfe, S. G. K. Tennakoon, L. Kondic, D. G. Schaeffer, and R. P. Behringer, "Granular friction, coulomb failure, and the fluid-solid transition for horizontally shaken granular materials," Phys. Rev. E 65, 031302 (2002).

[62] C. Ness, R. Mari, and M. E. Cates, "Shaken and stirred: Random organization reduces viscosity and dissipation in granular suspensions," Sci. Adv. 4, eaar3296 (2018).

[63] I. Lifshitz and V. Slyozov, "The kinetics of precipitation from supersaturated solid solutions," J. Phys. Chem. Solids 19, 35-50 (1961). 